\title{
Comparison of the Interactions Between Anionic Surfactants and Polyacrylonitrile
}

\author{
Said Abdul Nour
}

Received: 26 May 2014 / Accepted: 30 September 2014 / Published online: 11 December 2014

(C) The Author(s) 2014. This article is published with open access at Springerlink.com

\begin{abstract}
Viscosity and specific electrical conductivity of polyacrylonitrile (PAN) in $N, N$-dimethylformamide (DMF) medium are studied in the presence and absence of anionic surfactants as sodium oleate, sodium dodecyl sulfate and palmitic. Critical micelle concentrations of these surfactants are determined in DMF medium. Thermodynamic functions $\left(\Delta H_{\mathrm{m}}^{\circ}, \Delta G_{\mathrm{m}}^{\circ}\right.$ and $\Delta S_{\mathrm{m}}^{\circ}$ ) of micelle formation of sodium dodecylbenzene sulfonate and sodium dodecyl sulfate are determined. Curves of X-ray diffraction and differential thermal analysis showed that the PAN samples containing SDS lead to the development of the crystalline structure of PAN, and the initiation temperature decreases of the cyclization reaction of nitrile groups of PAN by $8.5^{\circ} \mathrm{C}$, respectively.
\end{abstract}

Keywords Polyacrylonitrile · Viscosity · Conductivity · Fiber $\cdot$ Mechanical and structural properties $\cdot$ Surfactants

\section{Introduction}

Since the invention of PAN-based carbon fibers in the 1950s by Shindo and Watt have attracted the world's attention as high-performance materials [1]. The PAN used in the textile industry is considered copolymers and has hardness linear chains [2-4].

Viscosity of PAN solution plays a significant role in the studying of mechanical and structural properties of produced fiber. A study of the relationship between viscosity of PAN solutions and concentration in DMF medium shows strong associations of PAN molecules, and this is because of the interactions between dipoles [5-7].

S. Abdul Nour $(\varangle)$

Department of Chemistry, Faculty of Science,

University of Aleppo, Aleppo, Syria

e-mail: abdulnoursaid@ hotmail.com
Crystalline structure of PAN in the solid phase can be controlled by changing the properties of its solutions, such as viscosity and electrical conductivity and surface tension $[8,9]$.

The mechanical and structural properties of polymeric solutions are strongly affected in the presence of surfactants; because the effectiveness of interactions between macromolecules themselves is significantly affected by these substances. These surfactants also affect the shape and dimensions of crystals being formed, and the conditions under which they grow. Unfortunately, studies about properties of these surfactants in different organic media, and their interactions with polymers which are dissolve in organic media only as PAN are still few and rare. However, the surfactants may be essential when developing fiber spinning procedures that would allow the control of mechanical and structural properties [10-15].

There are different types of interactions between surfactants and polymers; We mention here, for example, an electrostatic interaction (Fig. 1) [16], a hydrophobic interaction (Fig. 2) [17] and an interaction which can be formed a hydrogen bonding [18].

The purpose of this study is to show the different effects of anionic surfactants on the mechanical and structural properties of PAN. The new of this research is a study the properties of PAN at different concentrations and temperatures, the colloidal properties of different types of surfactants in DMF medium and interactions between PAN and surfactants.

\section{Experimental}

\subsection{Materials}

Industrial fibers of PAN with a molecular weight 100,000 $\mathrm{g} \mathrm{mol}^{-1}$, containing the following monomers: Acrylonitrile 


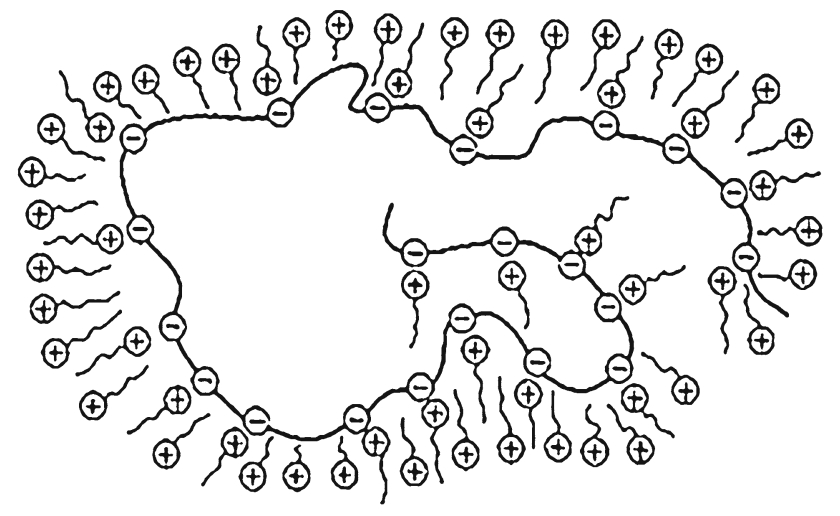

Fig. 1 Electrostatic interaction

$\mathrm{CH}_{2}=\mathrm{CH}-\mathrm{C} \equiv \mathrm{N} \quad(85 \mathrm{wt} \%) \quad \mathrm{w}, \quad$ Acryloamide $\mathrm{CH}_{2}=$ $\mathrm{CH}-\mathrm{CO}-\mathrm{NH}_{2}$ and Vinylacetate $\mathrm{CH}_{2}=\mathrm{CH}-\mathrm{O}-\mathrm{CO}-\mathrm{CH}_{3}$ (Adnan Shabarec Co). DMF solvent, $\mathrm{pH}$ value 7, purity (GC) $\geq 99.5 \%$ (MERCK Co). Sodium Oleate ("SO" $\mathrm{C}_{17} \mathrm{H}_{33}$ COONa) purity $95 \%$ (SIGMA Co). Sodium dodecyl sulfate ("SDS" $\mathrm{C}_{12} \mathrm{H}_{25} \mathrm{SO}_{4} \mathrm{Na}$ ) purity $\geq 99 \%$ (MERCK Co). Sodium dodecylbenzene sulfonate ("SDBS" $\mathrm{C}_{12} \mathrm{H}_{25} \mathrm{C}_{6} \mathrm{H}_{4}$ $\mathrm{SO}_{3} \mathrm{Na}$ ), (SIGMA Co). Palmitic acid ("PA" $\mathrm{C}_{15} \mathrm{H}_{31} \mathrm{COOH}$ ) purity $\geq 98 \%$ (MERCK Co).

\subsection{Instruments}

\subsubsection{Differential Thermal Analysis}

The differential thermal analysis (DTA) is carried out in Linseis PT-1600 at heating rate of $10^{\circ} \mathrm{C} \mathrm{min}^{-1}$ from ambient temperature to $400^{\circ} \mathrm{C}$ in a Helium environment.

\subsubsection{X-ray Diffractometer}

The patterns of X-ray diffraction (XRD) of PAN are obtained using a STOE transmission STADI diffractometer, with copper as target and nickel as filter $(\lambda=1.5408 \AA)$ at $30 \mathrm{kV}$ and $30 \mathrm{~mA}$. The scanning speed was $3.7 \mathrm{~min}^{-1}$ in the range $2 \theta=10^{\circ}-90^{\circ}$ at $298 \mathrm{~K}$.

\subsubsection{Conductometer}

Specific electrical conductivity is measured by SANXINMP 513 Lab Conductivity Meter.

\subsubsection{Falling Ball Viscometer}

The falling ball viscometer (HAAKE Co.) corresponds to the requirements of many international standards (ISO 12058 and the German standard DIN 53015). The measuring tube is jacketed by means of an outer glass tube, to control the solution temperature $\left( \pm 0.03^{\circ} \mathrm{C}\right.$ for test temperatures between 10 up to $80^{\circ} \mathrm{C}$ ). This device can measure the viscosity ranging between $0.3-75,000 \mathrm{mPa}$ s. Every value was measured three times and then averaged.

\subsection{Preparation of PAN Solutions}

PAN solutions are prepared in range $0.5-10 \mathrm{wt} \%$. PAN fibers are dissolved in DMF by continuous stirring at laboratory temperature, and then, transparent polymeric solutions are obtained. Also, PAN solutions are prepared in the presence of different concentrations of surfactant. Then, viscosity and Specific electrical conductivity of prepared solutions are studied.

Fig. 2 Hydrophobic interaction

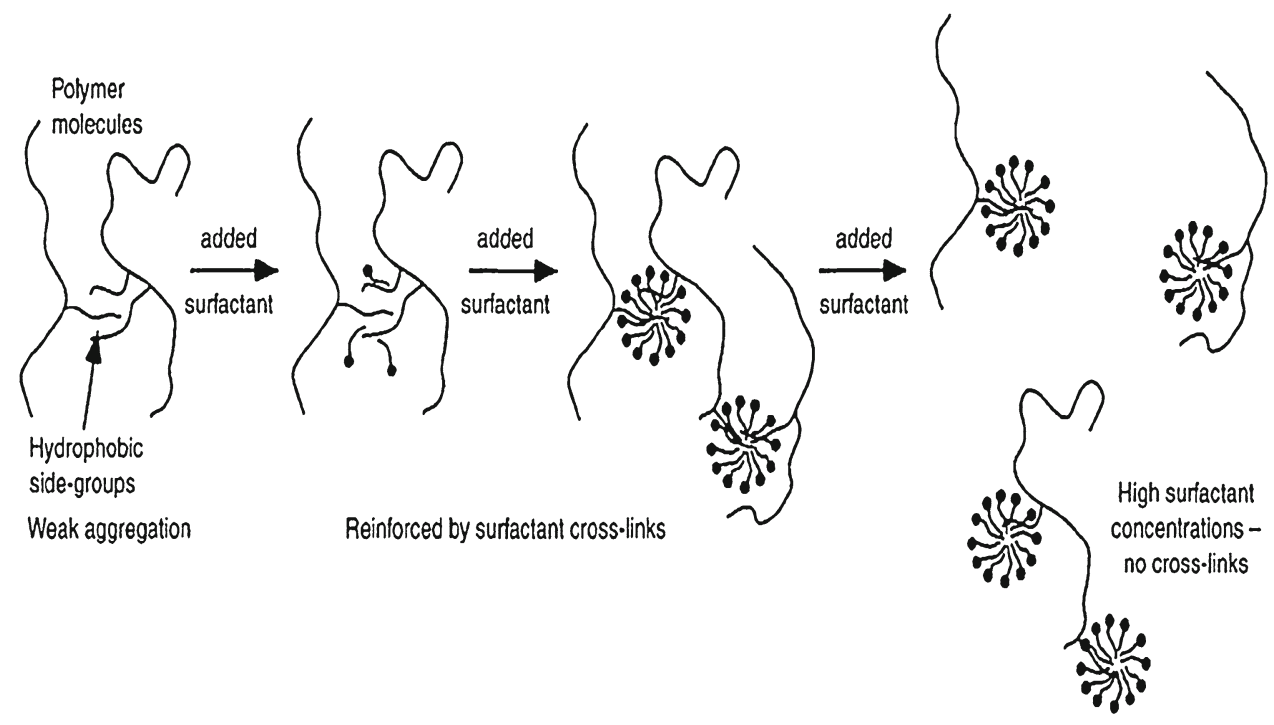




\subsection{Preparation of PAN Solid Samples}

The solid samples of PAN are prepared in order to study their structural and thermal properties in the following way. PAN solutions are prepared in the presence of surfactant and its absence, then poured in Petri dishes, put in dryer at $50^{\circ} \mathrm{C}$ until the complete vaporization of DMF, where solid samples are obtained, these samples are examined by XRD and DTA.

\section{Results and Discussion}

Viscosity $(\eta)$ of PAN solutions are measured at 298, 308, $318,328 \mathrm{~K}$, and specific electrical conductivity (SEC) is measured at 298, $308 \mathrm{~K}$. Viscosity of PAN solutions is estimated through the following equation:

$\eta=\kappa\left(d_{1}-d_{2}\right) t$

where $K$ ball constant, $d_{1}$ ball density, $d_{2}$ solution density, $t$ falling time of the ball.

Then, a specific viscosity $\left(\eta_{\mathrm{sp}}\right)$ calculated through the following equation [6]: $\eta_{\mathrm{sp}}=\eta-\eta_{\mathrm{o}} / \eta_{\circ}$

$\eta_{0}$ solvent viscosity.

Tables 1, 2 and 3 show the results of measuring the viscosity and the SEC respectively.

Figures 3 and 4 show the dependence of specific viscosity and SEC on PAN concentration, respectively.

Figure 3 shows that $\eta_{\mathrm{sp}}=f(C)$ is an exponential relationship. Specific viscosity increases in an almost straight line

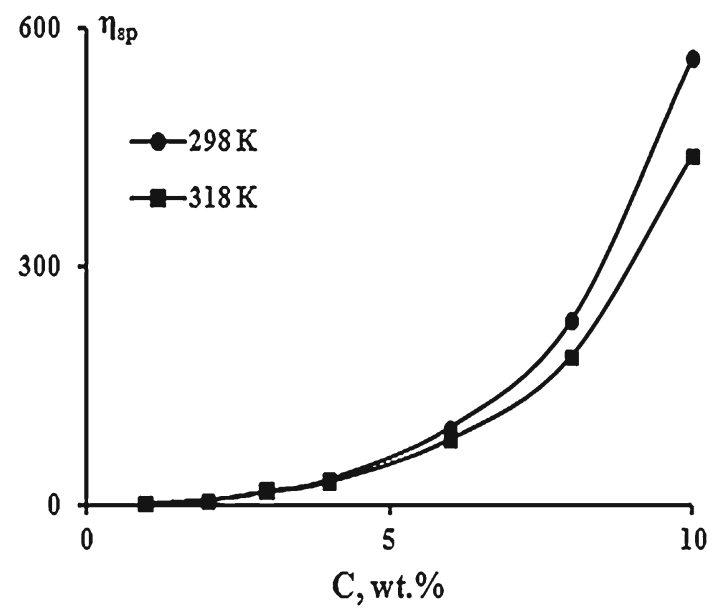

Fig. 3 Specific viscosity dependence on PAN concentration at 298, $318 \mathrm{~K}$

Table 1 Viscosity of PAN solutions

\begin{tabular}{|c|c|c|c|c|c|c|c|c|}
\hline \multirow[t]{3}{*}{$T(\mathrm{~K})$} & \multicolumn{8}{|c|}{ PAN concentration $C(\mathrm{wt} \%)$} \\
\hline & 0 & 1 & 2 & 3 & 4 & 6 & 8 & 10 \\
\hline & \multicolumn{8}{|c|}{ PAN viscosity $\eta$ (mPas) } \\
\hline 298 & 0.473 & 1.495 & 3.210 & 8.831 & 15.813 & 46.338 & 109.333 & 264.968 \\
\hline 308 & 0.420 & 1.221 & 2.751 & 7.359 & 12.876 & 37.971 & 90.210 & 224.973 \\
\hline 318 & 0.375 & 1.097 & 2.293 & 6.227 & 10.843 & 30.892 & 70.102 & 164.980 \\
\hline 328 & 0.339 & 0.955 & 2.030 & 5.321 & 9.261 & 25.743 & 56.234 & 129.980 \\
\hline
\end{tabular}

Table 2 specific viscosity of PAN solutions

\begin{tabular}{|c|c|c|c|c|c|c|c|}
\hline \multirow[t]{3}{*}{$T(\mathrm{~K})$} & \multicolumn{7}{|c|}{ PAN concentration $C(\mathrm{wt} \%)$} \\
\hline & 1 & 2 & 3 & 4 & 6 & 8 & 10 \\
\hline & \multicolumn{7}{|c|}{ Specific viscosity $\eta_{\mathrm{sp}}$} \\
\hline 298 & 2.160 & 5.786 & 17.670 & 32.431 & 96.966 & 230.147 & 559.186 \\
\hline 308 & 1.907 & 5.547 & 16.521 & 29.657 & 89.407 & 213.785 & 534.650 \\
\hline 318 & 1.925 & 5.114 & 15.605 & 27.914 & 81.378 & 185.938 & 438.946 \\
\hline 328 & 1.817 & 4.988 & 14.696 & 26.318 & 74.938 & 164.882 & 382.421 \\
\hline
\end{tabular}

Table 3 SEC of PAN solutions

\begin{tabular}{|c|c|c|c|c|c|c|c|c|c|}
\hline \multirow[t]{3}{*}{$T(\mathrm{~K})$} & \multicolumn{9}{|c|}{ PAN concentration $C(\mathrm{wt} \%)$} \\
\hline & 0 & 0.5 & 1 & 2 & 3 & 4 & 6 & 8 & 10 \\
\hline & \multicolumn{9}{|c|}{ PAN conductivity $\chi\left(\mu \mathrm{S} \mathrm{cm}^{-1}\right)$} \\
\hline 298 & 0.7 & 12.3 & 23.0 & 39.1 & 52.8 & 64.0 & 82.6 & 92.1 & 97.5 \\
\hline 308 & 0.8 & 14.5 & 27.8 & 44.0 & 59.8 & 70.9 & 88.9 & 102.0 & 111.4 \\
\hline
\end{tabular}




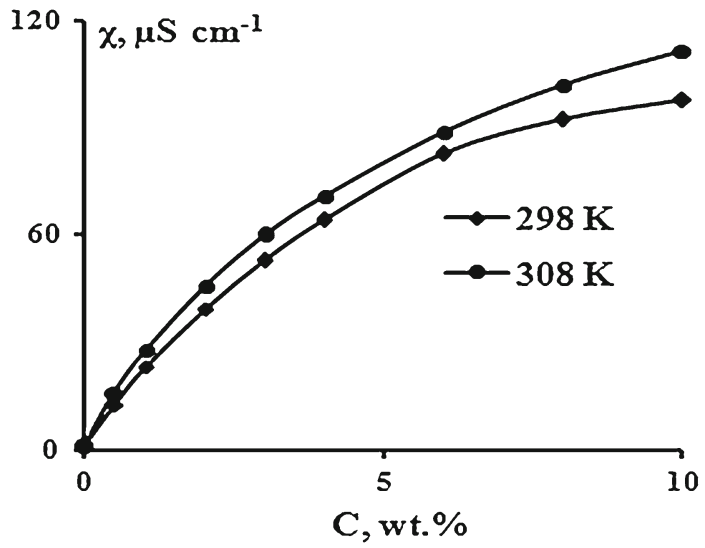

Fig. 4 SEC dependence on PAN concentration at 298, 308 K

at low concentrations, and then markedly increases starting with the percolation threshold of a crystalline structural formation ( $5 \mathrm{wt} \%$ approximately). This refers to the interaction between dipoles of PAN which leads to the occurrence of strong associations of macromolecules. Figure 3 also shows that temperature change does not affect the mechanism of crystalline structure formation; this may be due to the hardness of polymer chains $[2,6,9]$.

Figure 4 shows that $\chi=f(C)$ is a logarithmic relationship. SEC increases with increasing of PAN concentration, then after a concentration of $5 \mathrm{wt} \%$, conductivity becomes practically independent of concentration. This happens because of the completion of crystal structure [19].

The CMCs of anionic surfactants are determined by the SEC in DMF medium at $298 \mathrm{~K}$. The results are listed in Table 4.

The CMC of anionic surfactant in DMF medium is determined by using the following equation $\log \chi=f$ (c). Figure 5 shows that.

Figure 5 shows that the SEC $(\log \chi)$ versus concentration of anionic surfactants (C) plots show a break at a concentration corresponding to the CMC. Figure 5 also shows that the SEC increases at the beginning of the curve sharply, until the start of micelles formation then slowdown. This may be
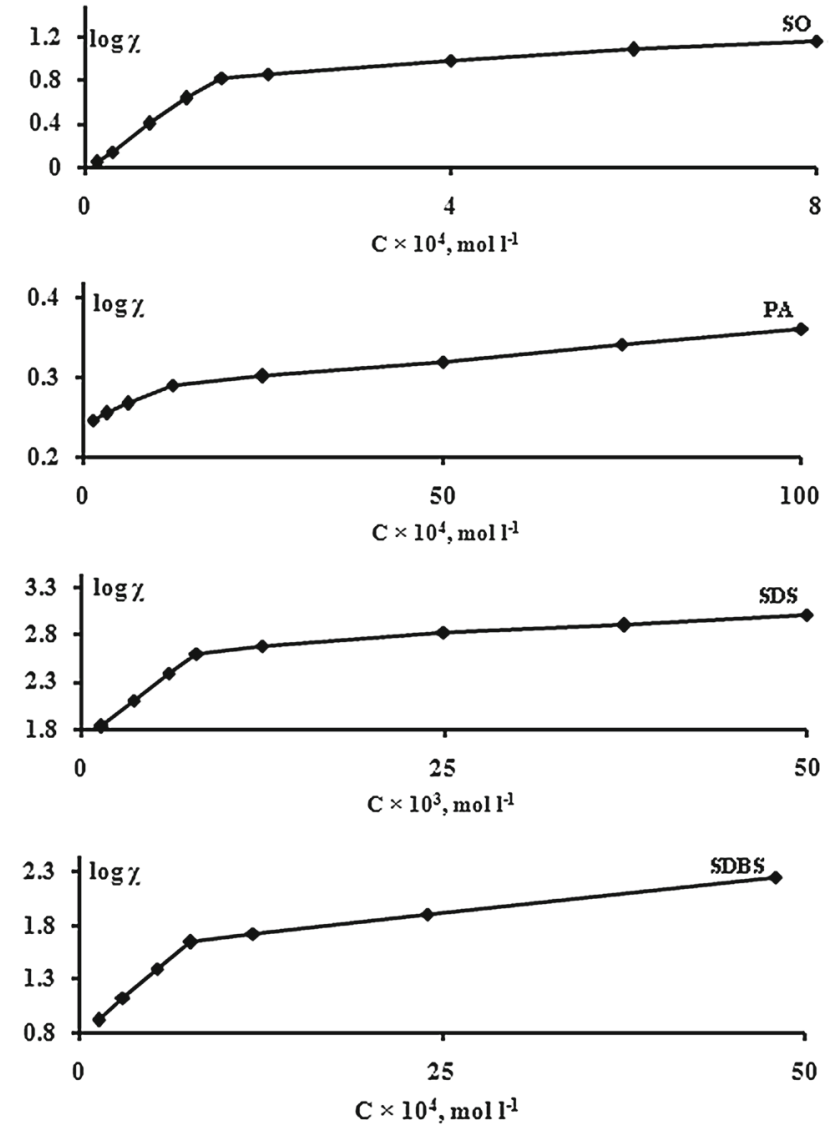

Fig. $5 \log \chi$ dependence on the anionic surfactant concentration

due to the partial link of counter ions with ionized micelles $[13,20,21]$.

Values of CMCs are listed in Table 5.

Table 5 Values of CMCs of anionic surfactants at $298 \mathrm{~K}$

\begin{tabular}{ll}
\hline Surfactant & $\mathrm{CMC} \times 10^{4}\left(\mathrm{moll}^{-1}\right)$ \\
\hline $\mathrm{SO}\left(\mathrm{C}_{17} \mathrm{H}_{33} \mathrm{COONa}\right)$ & 1.6 \\
PA $\left(\mathrm{C}_{15} \mathrm{H}_{31} \mathrm{COOH}\right)$ & 12.5 \\
$\mathrm{SDS}\left(\mathrm{C}_{12} \mathrm{H}_{25} \mathrm{SO}_{4} \mathrm{Na}\right)$ & 80 \\
SDBS $\left(\mathrm{C}_{12} \mathrm{H}_{25} \mathrm{C}_{6} \mathrm{H}_{4} \mathrm{SO}_{3} \mathrm{Na}\right)$ & 7.7 \\
\hline
\end{tabular}

Table 4 SEC of anionic surfactants solutions at different concentrations

\begin{tabular}{|c|c|c|c|c|c|c|c|}
\hline \multicolumn{2}{|l|}{ SO solutions } & \multicolumn{2}{|l|}{ PA solutions } & \multicolumn{2}{|l|}{$\underline{\text { SDS solutions }}$} & \multicolumn{2}{|l|}{ SDBS solutions } \\
\hline $\mathrm{C} \times 10^{4}\left(\mathrm{moll}^{-1}\right)$ & $\chi\left(\mu \mathrm{S} \mathrm{cm}^{-1}\right)$ & $\mathrm{C} \times 10^{4}\left(\mathrm{moll}^{-1}\right)$ & $\chi\left(\mu \mathrm{Scm}^{-1}\right)$ & $\mathrm{C} \times 10^{3}\left(\mathrm{moll}^{-1}\right)$ & $\chi\left(\mu \mathrm{S} \mathrm{cm}^{-1}\right)$ & $\mathrm{C} \times 10^{4}\left(\mathrm{moll}^{-1}\right)$ & $\chi\left(\mu \mathrm{S} \mathrm{cm}^{-1}\right)$ \\
\hline 0.12 & 1.1 & 1.56 & 1.77 & 1.56 & 67.3 & 1.5 & 8.2 \\
\hline 0.25 & 1.4 & 3.12 & 1.80 & 3.12 & 128.5 & 3.0 & 13.3 \\
\hline 0.50 & 2.5 & 6.25 & 1.84 & 6.25 & 247 & 6.0 & 24.5 \\
\hline 1.00 & 4.3 & 12.50 & 1.95 & 12.50 & 465 & 7.7 & 43.7 \\
\hline 2.00 & 7.1 & 25.00 & 2.00 & 25.00 & 631 & 12.0 & 48.7 \\
\hline 4.00 & 9.5 & 50.00 & 2.09 & 37.50 & 794 & 24.0 & 89.6 \\
\hline 6.00 & 11.9 & 75.00 & 2.20 & 50.00 & 1,023 & 48.0 & 176.0 \\
\hline 8.00 & 14.3 & 100.00 & 2.30 & - & - & - & - \\
\hline
\end{tabular}


Table 5 shows that CMC value of SDBS lower by about ten times that of SDS, which both has the same of dodecyl group $\left(\mathrm{C}_{12} \mathrm{H}_{25}\right)$. This is due to a link of benzene group which has a strong hydrophobic nature with the dodecyl group of SDBS. The disparity in the value of CMC for each of SO and PA is attributable to the difference of the chemical composition.

Thermodynamic functions of micelle formation of SDS and SDBS are studied because they have the same dodecyl group. The CMCs for each of them in DMF are identified at different temperatures.

CMCs values of SDS and SDBS at different temperatures are listed in Table 6.

Table 6 shows that with increasing temperature the CMC values of SDS and SDBS increase. This happens because when temperature increases the solubility of surfactant increases, therefore formation of surfactant micelle needs to a greater concentration.

The effect of temperature on micelle formation was investigated through the following Arrhenius equation:

$\mathrm{CMC}=A \exp \Delta H_{\mathrm{m}}^{\circ} / R T$

$\Delta H_{\mathrm{m}}^{\circ}$ enthalpy of micelle formation, $A$ constant related to the nature of the solution, $R$ universal gas constant, $T$ absolute temperature.

Equation (3) can be expressed in the logarithm form as:

Table 6 Values of CMCs of SDS and SDBS

\begin{tabular}{lll}
\hline$T(\mathrm{~K})$ & \multicolumn{2}{l}{$\mathrm{CMC} \times 10^{4}\left(\mathrm{moll}^{-1}\right)$} \\
\cline { 2 - 3 } & SDS & SDBS \\
\hline 298 & 7.7 & 80 \\
303 & 8.2 & 97 \\
308 & 9.0 & 110 \\
\hline
\end{tabular}

$\log \mathrm{CMC}=\log A+\frac{\Delta H_{\mathrm{m}}^{\circ}}{2.303 R} \frac{1}{T}$

The relationship (4) takes the following format in case we use anionic surfactants [22]:

$\log \mathrm{CMC}=\log A+\frac{\Delta H_{\mathrm{m}}^{\circ}}{4.606 R} \frac{1}{T}$

The plot of $\log \mathrm{CMC}$ against $1 / T$ yields a straight line, the slope of which gives $\left(\Delta H_{\mathrm{m}}^{\circ}\right)$. Then, Gibbs energy $\left(\Delta G_{\mathrm{m}}^{\circ}\right)$ and entropy $\left(\Delta S_{\mathrm{m}}^{\circ}\right)$ of micelle formation are determined from the following equations at $298 \mathrm{~K}$, respectively [23]:

$$
\begin{aligned}
\Delta G_{\mathrm{m}}^{\circ} & =2.303 R T \log \mathrm{CMC} \\
\Delta S_{\mathrm{m}}^{\circ} & =\frac{\Delta H_{\mathrm{m}}^{\circ}-\Delta G_{\mathrm{m}}^{\circ}}{T}
\end{aligned}
$$

Values of thermodynamic functions are listed in Table 7.

Table 7 shows that $\Delta G_{\mathrm{m}}^{\circ}<0$ is compatible with the fact that operation of micelle formation is spontaneous, $\Delta S_{\mathrm{m}}^{\circ}<0$ is because that a system becomes more regular when micelle formation. Also, we find that the leading force for the process of micelle formation is decrease of enthalpy. Additionally, Table 7 shows that decrease of entropy of SDS larger by six times than decrease of entropy of SDBS. This is due to the fact that hydrocarbon root length of SDS is shorter and therefore greater regularity of the system occurs when micelles are formed [12,23].

In order to investigate the effect of anionic surfactants on the hydrodynamic properties of PAN, we studied the viscosity and specific electrical conductivity of PAN solutions containing surfactants.

The results of measuring the viscosity of PAN solutions $1,10 \mathrm{wt} \%$ containing different concentrations of anionic surfactants at $298 \mathrm{~K}$ are showed in Tables 8 and 9, respectively.

Table 7 Values of thermodynamic functions

\begin{tabular}{llll}
\hline Material & $-\Delta H_{\mathrm{m}}^{\circ}\left(\mathrm{kJ} \mathrm{mol}^{-1}\right)$ & $-\Delta G_{\mathrm{m}}^{\circ}\left(\mathrm{kJ} \mathrm{mol}^{-1}\right)$ & $-\Delta S_{\mathrm{m}}^{\circ}\left(\mathrm{kJ} \mathrm{mol} \mathrm{K}^{-1}\right)$ \\
\hline $\mathrm{SDS}\left(\mathrm{C}_{12} \mathrm{H}_{25} \mathrm{SO}_{4} \mathrm{Na}\right)$ & 49 & 12 & 0.12 \\
$\mathrm{SDBS}\left(\mathrm{C}_{12} \mathrm{H}_{25} \mathrm{C}_{6} \mathrm{H}_{4} \mathrm{SO}_{3} \mathrm{Na}\right)$ & 24 & 18 & 0.02 \\
\hline
\end{tabular}

\begin{tabular}{|c|c|c|c|c|c|}
\hline \multicolumn{2}{|l|}{$\underline{\text { SO solutions }}$} & \multicolumn{2}{|l|}{$\underline{\text { PA solutions }}$} & \multicolumn{2}{|l|}{$\underline{\text { SDS solutions }}$} \\
\hline $\mathrm{C} \times 10^{4}\left(\mathrm{moll}^{-1}\right)$ & $\eta(\mathrm{mPas})$ & $\mathrm{C} \times 10^{4}\left(\mathrm{moll}^{-1}\right)$ & $\eta(\mathrm{mPas})$ & $\mathrm{C} \times 10^{3}\left(\mathrm{moll}^{-1}\right)$ & $\eta$ (mPas) \\
\hline 0.12 & 1.636 & 1.56 & 1.481 & 1.56 & 1.531 \\
\hline 0.25 & 1.004 & 3.12 & 1.477 & 3.12 & 1.534 \\
\hline 0.50 & 1.009 & 6.25 & 1.486 & 6.25 & 1.523 \\
\hline 1.00 & 0.995 & 12.50 & 1.415 & 12.50 & 1.516 \\
\hline 2.00 & 0.977 & 25.00 & 1.482 & 25.00 & 1.502 \\
\hline 4.00 & 0.986 & 50.00 & 1.433 & 37.50 & 1.507 \\
\hline 6.00 & 0.945 & 75.00 & 1.451 & 50.00 & 1.506 \\
\hline 8.00 & 0.935 & 100.00 & 1.477 & - & - \\
\hline
\end{tabular}

Table 8 Viscosity of PAN solutions 1 wt \% containing different concentrations of anionic surfactants 
Table 9 Viscosity of PAN solutions $10 \mathrm{wt} \%$ containing different concentrations of anionic surfactants

\begin{tabular}{|c|c|c|c|c|c|}
\hline \multicolumn{2}{|l|}{ SO solutions } & \multicolumn{2}{|l|}{ PA solutions } & \multicolumn{2}{|l|}{ SDS solutions } \\
\hline $\mathrm{C} \times 10^{4}\left(\mathrm{moll}^{-1}\right)$ & $\overline{\eta(\mathrm{mPas})}$ & $\mathrm{C} \times 10^{4}\left(\mathrm{moll}^{-1}\right)$ & $\overline{\eta(\mathrm{mPas})}$ & $\mathrm{C} \times 10^{3}\left(\mathrm{moll}^{-1}\right)$ & $\overline{\eta(\mathrm{mPas})}$ \\
\hline 0.12 & 267.320 & 1.56 & 271.052 & 1.56 & 304.963 \\
\hline 0.25 & 270.345 & 3.12 & 274.967 & 3.12 & 274.967 \\
\hline 0.50 & 272.332 & 6.25 & 273.865 & 6.25 & 254.969 \\
\hline 1.00 & 255.326 & 12.50 & 274.977 & 12.50 & 174.979 \\
\hline 2.00 & 155.198 & 25.00 & 269.768 & 25.00 & 159.980 \\
\hline 4.00 & 144.211 & 50.00 & 289.965 & 37.50 & 160.121 \\
\hline 6.00 & 144.533 & 75.00 & 287.125 & 50.00 & 164.980 \\
\hline 8.00 & 145.204 & 100.00 & 284.965 & - & - \\
\hline
\end{tabular}
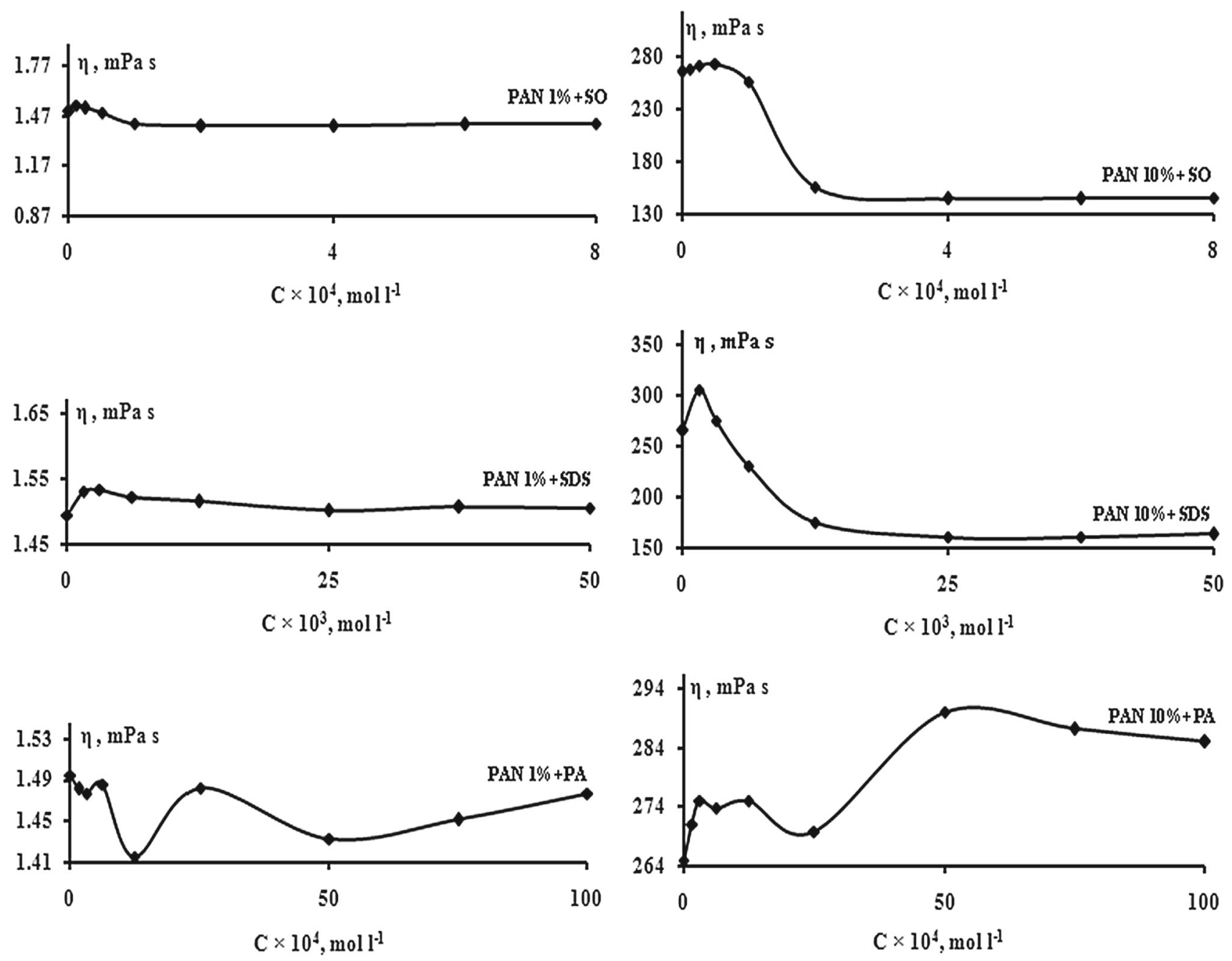

Fig. 6 Viscosity dependence of PAN solutions $1,10 \mathrm{wt} \%$ on the anionic surfactant concentration

Figure 6 shows the viscosity dependence of PAN solutions $1,10 \mathrm{wt} \%$ on the anionic surfactant concentration.

Figure 6 shows that the viscosity of PAN solutions 1, 10 wt $\%$ in the presence of SO, SDS and PA, starts decreasing at the field of CMC for each of them, but this decline will be sharp for both solutions PAN $(10 \%+$ SO) and PAN $(10 \%+$ SDS $)$. Whereas the effect of SO and SDS on the viscosity of PAN solutions (1 wt $\%)$ is almost insignificant. Also, from Fig. 6 we find that the relationship between viscosity of PAN solutions and PA concen- 
tration becomes complicated; the reason of this may be due to an acidity characteristic of PA and possibility of forming Louis salts with DMF. We conclude from above that effectiveness of the effect of anionic surfactants on the viscosity is determined by the concentration of polymer significantly $[7,17]$.

The results of measuring the SEC of PAN solutions 1, $10 \mathrm{wt} \%$ containing different concentrations of anionic surfactants at $298 \mathrm{~K}$ are showed in Tables 10 and 11 , respectively.

Figure 7 shows the SEC dependence of PAN solutions 1, $10 \mathrm{wt} \%$ on the anionic surfactant concentration.

Figure 7 shows that the SEC of PAN solutions 1, $10 \mathrm{wt} \%$ in the presence of SO and SDS increases at the beginning of the curve until the start of CMC then slow down, thus the conductivity becomes practically constant at the large concentrations for each of the SO and SDS. Additionally, conductivity of PAN/SO solutions increases with increasing of PAN concentration at all concentrations of SO, whereas conductivity of PAN/SDS solutions decreases with increasing of PAN concentration and this just at concentrations of SDS higher than CMC. Decrease of conductivity of the system (PAN 10\% + SDS) from conductivity of the system (PAN $1 \%+\mathrm{SDS}$ ) due to the occurrence of more associative points between PAN and SDS in the first system. This is because of the large concentration of PAN that leads to a decrease in the concentration of SDS micelles capable of transmitting electrical current. Figure 7 also shows that the presence of
PA in the PAN solutions $1,10 \mathrm{wt} \%$ does not affect on the conductivity significantly [17,24].

Structural and thermal properties of PAN in the presence of SDS are studied, because of its efficient effect on both vis-
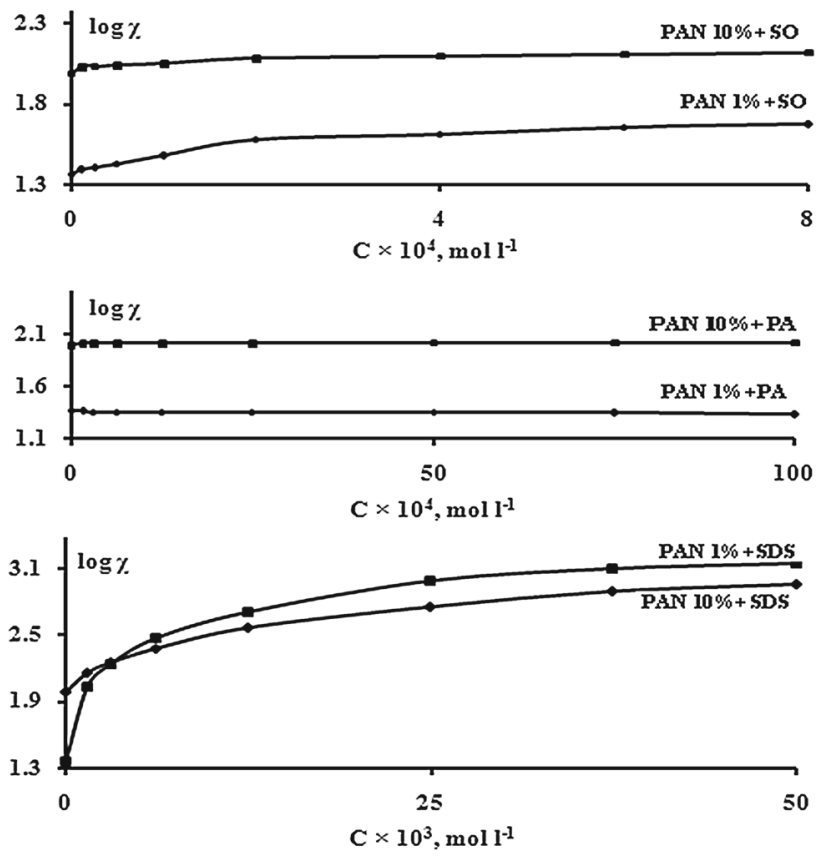

Fig. 7 SEC dependence of PAN solutions 1, 10wt \% on the anionic surfactant concentration

Table 10 SEC of PAN solutions $1 \mathrm{wt} \%$ containing different concentrations of anionic surfactants

\begin{tabular}{|c|c|c|c|c|c|}
\hline \multicolumn{2}{|l|}{ SO solutions } & \multicolumn{2}{|l|}{ PA solutions } & \multicolumn{2}{|l|}{ SDS solutions } \\
\hline $\mathrm{C} \times 10^{4}\left(\mathrm{~mol} \mathrm{l}^{-1}\right)$ & $\chi\left(\mu \mathrm{S} \mathrm{cm}^{-1}\right)$ & $\mathrm{C} \times 10^{4}\left(\mathrm{~mol} \mathrm{l}^{-1}\right)$ & $\chi\left(\mu \mathrm{S} \mathrm{cm}^{-1}\right)$ & $\mathrm{C} \times 10^{3},\left(\mathrm{~mol} \mathrm{l}^{-1}\right)$ & $\chi,\left(\mu \mathrm{S} \mathrm{cm}^{-1}\right)$ \\
\hline 0.12 & 24.4 & 1.56 & 22.7 & 1.56 & 107.9 \\
\hline 0.25 & 25.0 & 3.12 & 22.4 & 3.12 & 174.8 \\
\hline 0.50 & 26.8 & 6.25 & 22.3 & 6.25 & 294.9 \\
\hline 1.00 & 29.9 & 12.50 & 22.4 & 12.50 & 496.7 \\
\hline 2.00 & 38.2 & 25.00 & 22.5 & 25.00 & 975.0 \\
\hline 4.00 & 41.0 & 50.00 & 22.4 & 37.50 & 1257.9 \\
\hline 6.00 & 45.6 & 75.00 & 22.0 & 50.00 & 1391.0 \\
\hline 8.00 & 47.0 & 100.00 & 21.3 & - & - \\
\hline
\end{tabular}

Table 11 SEC of PAN solutions $10 \mathrm{wt} \%$ containing different concentrations of anionic surfactants

\begin{tabular}{|c|c|c|c|c|c|}
\hline \multicolumn{2}{|l|}{$\underline{\text { SO solutions }}$} & \multicolumn{2}{|l|}{ PA solutions } & \multicolumn{2}{|l|}{ SDS solutions } \\
\hline $\mathrm{C} \times 10^{4}\left(\mathrm{~mol} \mathrm{l}^{-1}\right)$ & $\chi\left(\mu \mathrm{S} \mathrm{cm}^{-1}\right)$ & $\mathrm{C} \times 10^{4}\left(\mathrm{~mol} \mathrm{l}^{-1}\right)$ & $\chi\left(\mu \mathrm{S} \mathrm{cm}^{-1}\right)$ & $\mathrm{C} \times 10^{3}\left(\mathrm{~mol} \mathrm{l}^{-1}\right)$ & $\chi\left(\mu \mathrm{S} \mathrm{cm}^{-1}\right)$ \\
\hline 0.12 & 107.0 & 1.56 & 101.1 & 1.56 & 143 \\
\hline 0.25 & 107.9 & 3.12 & 102.8 & 3.12 & 178 \\
\hline 0.50 & 109.6 & 6.25 & 103.3 & 6.25 & 236 \\
\hline 1.00 & 111.9 & 12.50 & 103.0 & 12.50 & 366 \\
\hline 2.00 & 117.2 & 25.00 & 103.5 & 25.00 & 570 \\
\hline 4.00 & 121.1 & 50.00 & 104.0 & 37.50 & 780 \\
\hline 6.00 & 126.7 & 75.00 & 104.0 & 50.00 & 912 \\
\hline 8.00 & 132.0 & 100.00 & 103.8 & - & - \\
\hline
\end{tabular}


Fig. 8 X-ray diffraction patterns. a PAN $10 \mathrm{wt} \%$, b PAN $10 \mathrm{wt} \%$ containing $25 \times 10^{-3} \mathrm{moll}^{-1}$ of SDS
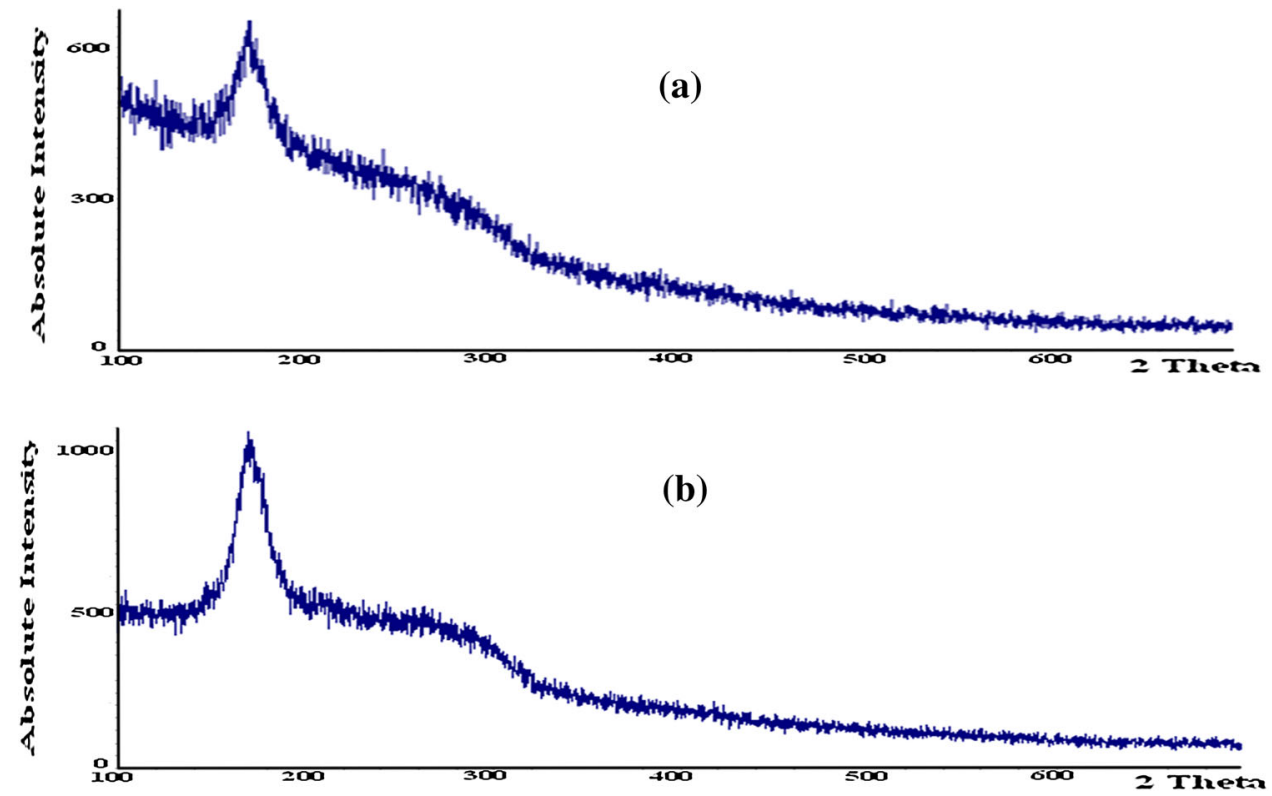

cosity and conductivity. Therefore, two solid samples were prepared, as described in the experimental section, the first one is PAN $10 \mathrm{wt} \%$, and the second one is PAN $10 \mathrm{wt} \%$ containing $25 \times 10^{-3} \mathrm{~mol} \mathrm{l}^{-1}$ of SDS. Then, these samples are tested by XRD and DTA. The X- ray diffractometer has software to evaluate crystalline structure. Figure 8 shows the Xray diffraction patterns.

Figure 8 shows that X-ray diffraction patterns have two peaks; the first one has a strong intensity, while the second is weak and broad, the first peak indicates to a crystalline structure, while the second peak indicates that there is still some kind of amorphous structure [25]. Figure $8 \mathrm{~b}$ also shows that the intensity of the first peak increases, in comparison with the first peak in Fig. 8a, which proves that the crystalline structure has developed in the presence of SDS.

Figure 9 shows the DTA curves.

Figure 9 shows that the DTA curve of two samples have an exothermic peak indicates to a cyclization of nitrile groups of PAN. Figure 9a shows that the initiation temperature of DTA curve started at $295.2^{\circ} \mathrm{C}$ with a peak maximum at $301.8^{\circ} \mathrm{C}$, while Fig. $9 \mathrm{~b}$ shows that the initiation temperature of DTA curve started at $286.7^{\circ} \mathrm{C}$ with a peak maximum at $297.6^{\circ} \mathrm{C}$, this means that the start of the cyclization reaction of nitrile groups of a sample containing SDS is a lower by $8.5^{\circ} \mathrm{C}$. Additionally, Fig. $9 \mathrm{~b}$ shows that the peak intensity is rises in comparison with the peak intensity in Fig. 9a; this refers to increasing the cyclization reaction of nitrile groups and regularity of PAN structure in the presence of SDS [26,27].

\section{Conclusions}

- We have found that the specific viscosity and the specific electrical conductivity dependence on PAN concentration is an exponential and logarithmic relationship, respectively, where the specific viscosity increases dramatically after the crystalline structural formation, whereas conductivity becomes practically independent of concentration after the crystalline structural formation.

- The CMC of anionic Surfactants (SO, PA, SDS and SDBS) in DMF medium has been determined.

- The leading force for the process of micelle formation of SDS and SDBS is the decrease of enthalpy.

- Effectiveness of the effect of SO, PA and SDS on viscosity is determined by the concentration of polymer significantly.

- Conductivity of PAN solutions is strongly influenced in the presence of SDS and SO, while conductivity of PAN solutions containing PA practically dose not affected.

- The foundations of changes of hydrodynamic properties of PAN solutions in the presence of SO, PA and SDS and its absence have been laid.

- We have found that the crystalline structure of PAN has developed in the presence of SDS.

- The initiation temperature decreases of the cyclization reaction of nitrile groups of PAN in the presence of SDS by $8.5^{\circ} \mathrm{C}$. 

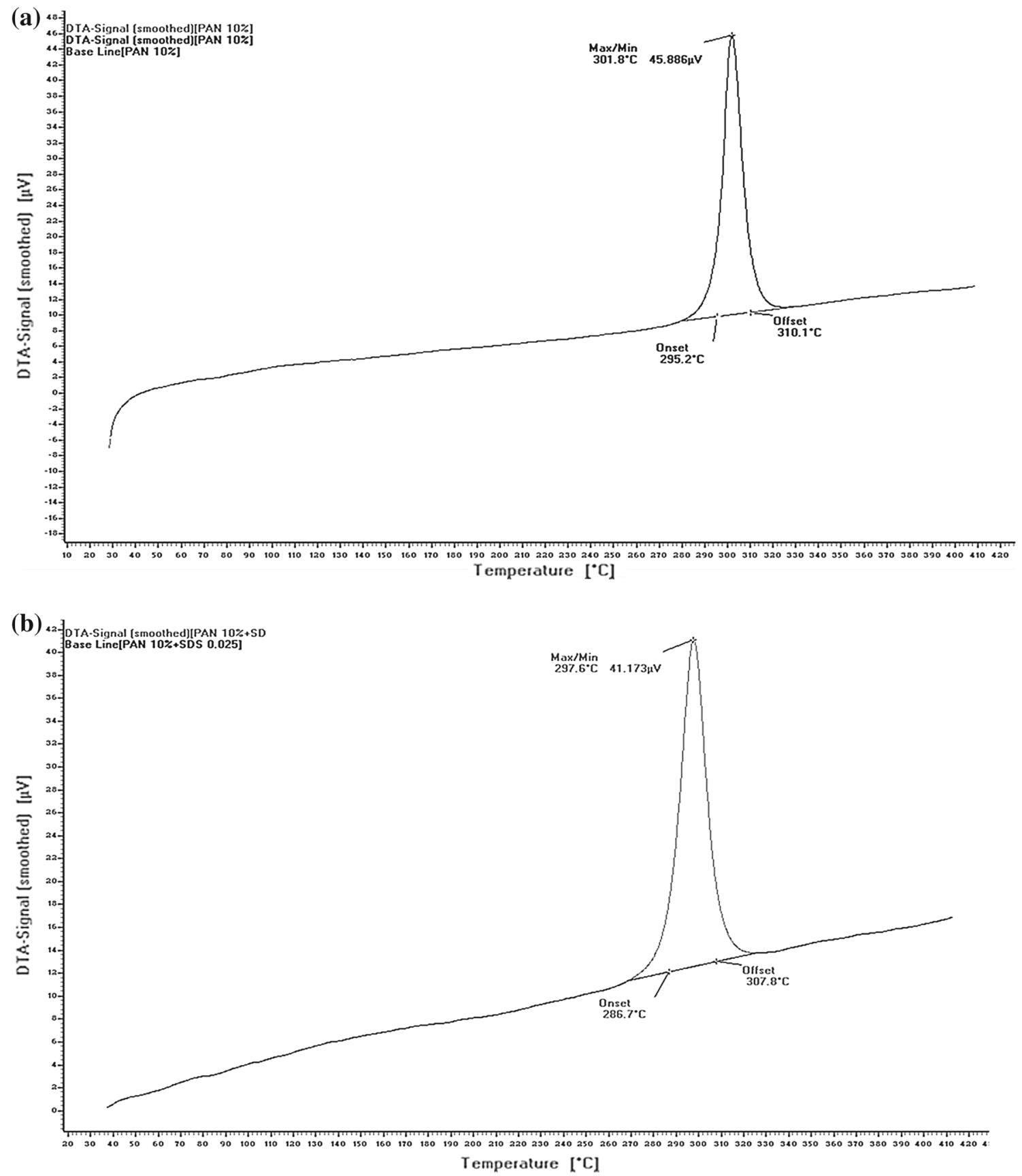

Fig. 9 DTA curves. a PAN $10 \mathrm{wt} \%$, b PAN $10 \mathrm{wt} \%$ containing $25 \times 10^{-3} \mathrm{moll}^{-1}$ of SDS

Acknowledgments The researcher greatly acknowledges the support he has received from the Atomic Energy Commission (SYR), Adnan Shabarec Co. (SYR), Dr. Bahjat Falazi, Dr. Nazeeh Kutaish, Dr. Nassoh Alaya, Dr. Samer Gharibe, Mr. Akram Yaghmour and Mr. Muhammad Alhameish.

Open Access This article is distributed under the terms of the Creative Commons Attribution License which permits any use, distribution, and reproduction in any medium, provided the original author(s) and the source are credited.

\section{References}

1. Qiang, X.; Sizhu, W.: A study on the orientation structure and mechanical properties of polyacrylonitrile precursors. Polym. Adv. Technol. 16, 642 (2005)

2. Alnajady, A.F.: Technology of Industrial Fibers and their Mixtures. Mounshaat Almaaref, Alexandria (1993)

3. Lodge, T.: Polymer Chemistry. Wood Head Publishing, London (2010) 
4. Morgan, P.: Carbon Fibers and Their Composites. Taylor \& Francis, New York (2005)

5. Liu, Q.; Xiong, M.: Preparation of branched polyacrylonitrile through self-condensing vinyl copolymerization. J. Appl. Polym. Sci. 110, 494 (2008)

6. Furukawa, J.: Physical Chemistry of Polymer Rheology. Springer, Tokyo (2010)

7. Abdul Nour, S.: Regulating of Supermolecular Structure of Polyacrylonitrile by Surfactants. Thesis, Ph.D. Thesis, Mendeleev University of Chemical Technology, Moscow (1990)

8. Liwen, J.; Medford, A.: Electrospun polyacrylonitrile/zing chloride composite nanofibers and their response to hydrogen sulfide. Polymer 50, 605 (2009)

9. Satish, K.; Tong, W.: Electrospinning of polyacrylonitrile nanofibers. J. Appl. Polym. Sci. 102, 1023 (2006)

10. Kamide, K.; Dobashi, T.: Physical Chemistry of Polymer Solutions. Elsevier Science, Amsterdam (2000)

11. Williams, G.R.; Bligh, S.W.: Coaxial electrospinning with sodium dodecylbenzene sulfonate solution for high quality polyacrylonitrile nanofibers. Colloids Surf. 396, 161 (2012)

12. Birdi, K.: Surface and Colloid Chemistry. CRC Press, New York (2010)

13. Voyutsky, S.: Colloid Chemistry. Mir Publisher, Moscow (1978)

14. Abdul Nour, S.: Study of the intermolecular interaction between polyacrylonitrile and amphoteric surfactant (betaine) in dimethylformamide. Asian J. Chem. 26, 4810 (2014)

15. Xia, W.; Deng, Y.: Coaxial Electrospinning with triton X-100 solutions as sheath fluids for preparing PAN nanofibers. Macromol. Mater. Eng. 297, 395 (2012)

16. Nagarjan, R.: On nature interaction between polymers and surfactants in dilute aqueous solutions. Am. Chem. Soc. 2, 33 (1981)

17. Odeh, F.: Polymer-Surfactant Interaction. Clarkson University, New York (2006)
18. Wang, D.: Compatibility studies with blends based on hydroxypropylcellulose and polyacrylonitrile. Carbohydr. Polym. 73, 191 (2008)

19. Pan, W.; Yang, S.: Relationship between electrical conductivity and phase morphology of polyaniline/polyacrylonitrile and polyaniline/polystyrene blends. Int. J. Polym. Mater. 54, 21 (2005)

20. Arani, M.; Bahrami, H.: Dynamic adsorption of gemini and conventional cationic surfactants onto polyacrylonitrile. Colloids Surf. 307, 121 (2007)

21. Falazy, B.: Colloid Chemistry. Aleppo University Publication, Aleppo (1992)

22. Kutaish, N.: Colloid Chemistry. Aleppo University Publication, Aleppo (1982)

23. Fralov, U.G.; Grodsky, A.S.: Laboratory Experiments and Problems in Colloid Chemistry. Mir Publisher, Moscow (1986)

24. Tong, P.; Dublin, P.: Colloid-Polymer Interaction. American Chemical Society, Washington (1993)

25. Xiao, H.; Shan Yuan, W.: Interior structure of polyacrylonitrile nanofibers with LiCl. Mater. Lett. 62, 1325 (2008)

26. Hatakeyama, T.; Quinn, F.: Thermal Analysis "Fundamentals and Applications to Polymer Science. 2nd edn. Wiley, England (1999)

27. Yong, X.; Meijie, Y.: Influence of precursor properties on the thermal stabilization of polyacrylonitrile fibers. Polym. Bull. 57, 757 (2006) 